\title{
Estratégias de aprendizagem utilizadas por meninos e meninas: Análise comparativa
}

\author{
Learning strategies used by boys and girls: A comparative analysis
}

\footnotetext{
[a] Universidade Federal do Rio Grande do Norte, Natal, RN Brasil, e-mail: manuela_rcl@ hotmail.com

${ }^{\left[{ }^{b]}\right.}$ Universidade Estadual da Paraíba, Campina Grande, PB - Brasil, e-mail:monillyramos@gmail.com

${ }^{[c]}$ Universidade Federal da Paraíba, João Pessoa, PB - Brasil, e-mail: carlamoita@globo.com
}

Recebido: 22/09/2011 Received: 09/22/2011

Aprovado: 04/10/2012 Approved: 10/04/2012

\author{
Manuela Ramos Caldas Lins ${ }^{[a]}$, Monilly Ramos Araujo ${ }^{[b]}$, Carla Alexandra da Silva Moita Minervino ${ }^{[c]}$
}

\section{Resumo}

A presente investigação teve como objetivo analisar diferenças no repertório de estratégias de aprendizagem utilizado por escolares dos sexos masculino e feminino, tendo em vista que pesquisas nacionais e internacionais têm demonstrado que as estratégias de aprendizagem, entendidas como os métodos utilizados pelos estudantes para adquirir conhecimento, estão diretamente relacionadas ao alto desempenho escolar e que a frequência no uso dessas estratégias varia conforme o sexo dos escolares. Participaram do estudo 68 estudantes, matriculados do $6^{\circ}$ ao $8^{\circ}$ ano do Ensino Fundamental de escolas públicas. 0 instrumento utilizado na coleta de dados foi uma escala de estratégias de aprendizagem. De modo geral, os resultados indicaram haver diferenças entre o desempenho de meninos e de meninas, embora essas não tenham sido estatisticamente significantes. Sugere-se que novas investigações sejam realizadas, com uma amostra mais representativa, a fim de examinar a diferença entre os sexos ao utilizarem estratégias de aprendizagem, buscando aprofundar o que influencia essa possível diferença.

Palavras-chave: Estratégias de Aprendizagem. Ensino Fundamental. Escolas Públicas.

\begin{abstract}
This investigation aimed to analyze differences in the repertoire of learning strategies used by male and female students, given that national and international studies have shown that learning strategies, understood as the methods used by students to acquire knowledge, are directly related to high performance in school and the frequency in use of these strategies varies according to the gender of the students. 68 students enrolled from the $6^{\text {th }}$ to $8^{\text {th }}$ grade in public elementary schools participated of this research. The instrument used for data collection was a scale of learning strategies. Overall, the results showed difference between the performance of boys and girls, although these were not statistically significant. It is suggested that further investigations are carried out with larger sampling to examine the difference between the genders use of learning strategies, seeking to deepen what influences this potential difference.
\end{abstract}

Keywords: Learning Strategies. Elementary School. Public Schools.

Psicol. Argum. 2014 jul./set., 32(78), 9-15 


\section{Introdução}

Atualmente, devido às exigências do mercado, a escolarização e a consequente qualificação profissional deixou de ser um ideal e passou a ser uma necessidade. Diante dessa demanda, a relação entre educandos, educadores e escola sofreu alterações de forma tal que os desafios enfrentados pelos professores para que a aprendizagem dos educandos se efetive parecem ter se intensificado. Nesse contexto, pesquisadores vêm trabalhando no sentido de analisar como tornar a aprendizagem mais eficaz, inclusive através do estudo dos métodos que os estudantes utilizam para apreender determinado assunto, as chamadas estratégias de aprendizagem, que podem ser compreendidas conforme aponta Dembo (1994) como métodos que os estudantes utilizam para adquirir conhecimento. De modo semelhante Silva e Sá (1997) definem as estratégias de aprendizagem, em seu nível mais complexo, como processos conscientes utilizados pelos estudantes para atingir os objetivos da aprendizagem e, em seu nível mais específico, como qualquer procedimento usado para realizar uma determinada tarefa. As autoras destacam que as estratégias são procedimentos controláveis, visto que o sujeito deve operar sobre elas de modo intencional.

Cinco tipos de estratégias de aprendizagem foram identificados por Weinstein e Mayer (1983), são elas: estratégias de ensaio (envolvem repetir ativamente o material a ser aprendido); estratégias de elaboração (implicam na realização de conexões entre o material novo a ser aprendido e o material antigo e familiar); estratégias de organização (referem-se à imposição de estrutura ao material a ser aprendido); estratégias de monitoramento (implicam que o indivíduo deve estar constantemente monitorando sua capacidade de captar e absorver o conteúdo que está sendo ensinado); e estratégias afetivas (referem-se à eliminação de sentimentos desagradáveis, que não condizem com a aprendizagem).

Diferentemente, Zimmerman e Martinez-Pons (1988) encontraram 14 tipos de estratégias, a saber: autoavaliação, organização e transformação, estabelecimento de metas e planejamento, busca de informação, registro de informação, automonitoramento, organização do ambiente, busca de ajuda e revisão. Dentre essas estratégias, Boruchovitch et al. (2006) pontuam que as mais utilizadas por alunos das mais diversas faixas etárias e nacionalidades são as de memorização, de elaboração, de administração de tempo, de estruturação do ambiente, de autoavaliação e de apoio afetivo.

As estratégias de aprendizagem podem ainda ser cognitivas ou metacognitivas. Para Dembo (1994), as estratégias cognitivas se referem a comportamentos e pensamentos que influenciam o processo de aprendizagem de maneira que a informação possa ser armazenada de forma mais eficiente. As estratégias metacognitivas, por sua vez, são procedimentos que o indivíduo usa para planejar, monitorar e regular seu próprio pensamento.

Independentemente da classificação utilizada, é consenso entre os pesquisadores da área, como aponta Boruchovitch $(1999 ;$ 2001), que um aluno com bom desempenho acadêmico é capaz de selecionar corretamente as estratégias de aprendizagem conforme o objetivo da atividade que precisa desempenhar e, além disso, é sempre capaz de modificar essas estratégias quando não são úteis, pois constantemente monitoram sua compreensão. Por essa razão, Ribeiro (2002) afirma que é importante que os alunos conheçam a estratégia, saibam como aplicá-la, monitorem sua eficácia, confrontem os resultados e os avaliem. Igualmente, Boruchovitch et al. (2006) garantem que, para que o aluno desenvolva suas capacidades cognitivas, faz-se necessário que, além de conhecer as estratégias de aprendizagem, ele saiba como e quando usá-las, demonstrando que a aprendizagem mecânica das estratégias de aprendizagem não é suficiente para que os estudantes as utilizem de forma eficaz e, portanto, melhorem seus resultados acadêmicos.

Nesse sentido, Silva e Sá (1997) afirmam que a instrução em estratégias de aprendizagem pode abrir novas perspectivas para uma potencialização da aprendizagem, tendo em vista que ensinar ao aluno técnicas para aprender melhor pode influenciar tanto a aprendizagem como a performance acadêmica. Corroborando, Costa (2000) salienta que o ensino de estratégias de aprendizagem, embora não seja condição suficiente para o sucesso acadêmico, faz-se necessário na medida que pode maximizar o desempenho do aluno.

Diversos instrumentos têm sido utilizados para avaliar as estratégias de aprendizagem de estudantes no país. Dentre eles destaca-se uma escala que foi desenvolvida por Boruchovitch e Santos, no ano de 2001. Tal escala, conforme aponta Oliveira 
(2008), apresentava inicialmente 40 itens fechados, em formato likert, e uma questão aberta que buscava levantar outras estratégias utilizadas pelos estudantes, mas que não haviam sido contempladas na escala.

Alguns pesquisadores fizeram uso dessa versão inicial da escala, dentre eles, Gomes (2002) e Gomes e Boruchovitch (2005), que aplicaram a escala em crianças do 5ำ ano do ensino fundamental, com os objetivos de adaptar um jogo para avaliar as estratégias de aprendizagem, verificar a existência de relações entre a compreensão da leitura e o desempenho no jogo e explorar relações entre as estratégias de aprendizagem e o desempenho no jogo. Participaram do estudo 29 estudantes, de ambos os sexos, de uma escola pública de uma cidade paulista. Dentre os resultados, apesar de não objetivar avaliar diferença entre os gêneros, as autoras identificaram que as meninas obtiveram uma melhor pontuação $(M=58,91)$ quando comparadas aos meninos $(M=55,50)$, evidenciando que elas utilizaram mais estratégias de aprendizagem do que eles.

Autoras como Cruvinel (2003) e Cruvinel e Boruchovitch (2004) também recorreram à escala de estratégias de aprendizagem de 40 itens, bem como a outros instrumentos, com o objetivo de verificar a relação entre sintomas depressivos, rendimento escolar e estratégias de aprendizagem em escolares do ensino fundamental. Para tanto, avaliaram 169 crianças, de ambos os sexos, do 4ํㅜㅇ 5음 e $6^{\circ}$ anos de uma escola pública, com nível social e econômico desfavorecido. Dentre os resultados, as autoras identificaram a existência de diferenças entre os sexos em favor das meninas, apesar dessa diferença não ser estatisticamente significante.

Utilizando-se de uma versão aprimorada da escala Oliveira (2008) e Oliveira, Boruchovitch e Santos (2011), com o objetivo de validar o instrumento, entrevistaram 815 estudantes, do $3^{\circ}$ ao $9^{\circ}$ anos do ensino fundamental, de ambos os sexos, de escolas públicas e privadas dos estados de São Paulo e Minas Gerais. Os dados demonstraram haver diferenças no uso das estratégias de aprendizagem quando considerado o sexo, de forma que as estudantes do sexo feminino alcançaram pontuações mais elevadas. Nesse caso, a diferença entre os sexos, inclusive, foi estatisticamente significante.

Com base nesses dados e com o intuito de analisar o repertório de estratégias de aprendizagem utilizado por escolares da cidade de Campina Grande, no interior do estado da Paraíba, Lins, Araujo e Minervino (2009; 2011) utilizaram-se da Escala de Estratégias de Aprendizagem na versão de 31 itens, validada por Oliveira, em 2008. Participaram do estudo 491 estudantes, do sexo masculino e do sexo feminino, matriculados do $6^{\circ}$ ao $9^{\circ}$ anos do Ensino Fundamental de escolas particulares e públicas, com idade média de 12 anos e 7 meses $(D P=1,7)$. De modo geral, os resultados confirmaram os estudos anteriores, visto que observou-se, dentre outros resultados, que o sexo feminino alcançou uma pontuação mais elevada do que o sexo masculino.

Diante desses resultados, pode-se pensar que as meninas utilizam mais estratégias de aprendizagem do que os meninos, embora não esteja claro em que direção essas diferenças ocorrem, se na frequência ou na quantidade de estratégias. Assim sendo, questiona-se: as meninas utilizam as mesmas estratégias que os meninos em proporção mais elevada ou elas utilizam estratégias diferenciadas que garantem ao final uma melhor pontuação? Visando contribuir com as pesquisas nacionais e possibilitar uma discussão mais profícua das estratégias é que o presente trabalho objetiva investigar as diferenças no repertório de estratégias de aprendizagem de meninos e meninas matriculados no Ensino Fundamental.

\section{Método}

\section{Aspectos éticos}

Para operacionalização da pesquisa, o projeto foi submetido à apreciação do Comitê de Ética em Pesquisa do Centro de Ciências Biológicas e da Saúde, da Universidade Estadual da Paraíba, que emitiu parecer favorável a sua realização sob a CAAE - 0546.0.133.000-08. A presente pesquisa respeitou os padrões éticos de conduta conforme a Resolução no 196/96 do Conselho Nacional de Saúde/MS.

\section{Participantes}

0 estudo foi realizado com 92 estudantes. Contudo, visando obter um número equivalente de estudantes do sexo masculino e do sexo feminino, sorteou-se 68 alunos tomando por base o sexo e a série escolar. Assim, o sexo feminino representou $50 \%(n=34)$ da amostra e o masculino 50\% $(n=34)$.

Psicol. Argum. 2014 jul./set., 32(78), 9-15 
Do total de participantes, $38,3 \%(n=26)$ eram do $6^{\circ}$ ano, $38,3 \%(n=26)$ do $7^{\circ}$ ano e $23,4 \%(n=16)$ do $8^{\circ}$ ano do Ensino Fundamental de escolas públicas de uma cidade do interior da Paraíba. A média de idade foi de 12 anos e 2 meses $(D P=1,5)$, sendo a idade máxima de 16 anos e a mínima de 9 anos.

Instrumento

O instrumento utilizado para a coleta de dados foi uma "Escala de Estratégias de Aprendizagem", de autoria de Boruchovitch e Santos, validada por Oliveira, em 2008, a qual se caracteriza por:

a) Ficha de identificação dos estudantes que se refere a um conjunto de questões relativas aos dados pessoais como nome, sexo, idade, escola, série e repetência dos participantes.

b) Dados relativos à utilização de estratégias de aprendizagem, contendo 31 questões, com três possibilidades de respostas para cada uma das questões. As categorias são descritas como: sempre, valendo 3 pontos; às vezes, 2 pontos; e nunca, valendo 1 ponto. Os itens pertencentes ao fator ausência de estratégias $(3,7,8,12,15,19,21,23,24,25,26,28$ e 30) têm sua pontuação invertida. A pontuação máxima possível é 93 pontos e a mínima 31 pontos.

\section{Procedimentos}

Inicialmente, foi realizado contato com as escolas, para expor a proposta de trabalho, com a direção e/ou a coordenação. Os responsáveis pelas instituições que concordaram com a realização da pesquisa em suas dependências assinaram o Termo de Consentimento Institucional. Posteriormente, após esclarecimentos sobre a pesquisa aos estudantes, foi solicitado o consentimento dos pais e/ ou responsáveis através da assinatura do Termo de Consentimento Livre e Esclarecido (TCLE).

Em horário previamente agendado com os diretores das instituições, compareceu-se às escolas e os estudantes que estavam com o TCLE devidamente assinado e que desejaram participar responderam o instrumento. Após um breve rapport, os objetivos da pesquisa foram retomados, e foi informado aos estudantes que a atividade não valia nota. Em seguida, o aplicador orientou as crianças sobre o preenchimento do cabeçalho do instrumento, leu o primeiro item, aguardou um tempo para que todos respondessem, em seguida perguntou se todos haviam entendido a tarefa e diante da confirmação, pediu que os estudantes prosseguissem sozinhos.

A aplicação ocorreu de forma coletiva e, de forma geral, durou 20 minutos em cada sala de aula. Em todas as ocasiões, os professores, que estavam ministrando alguma disciplina no momento da aplicação, permaneceram no local acompanhando a atividade.

\section{Análise de resultados}

Os dados foram tabulados com o auxílio do pacote estatístico Statistical Package for the Social Sciences (SPSS), versão 17 for Windows e submetidos às estatísticas descritiva e inferencial, visando atender os objetivos propostos. Cabe ressaltar que a distribuição estatística da população apresentou uma curva normal, utilizou testes paramétricos e o nível de significância adotado foi de 5\%.

Inicialmente, buscou-se investigar a média obtida na escala de estratégias de aprendizagem pelos participantes. Os meninos alcançaram uma média de 67,6 $(D P=5,9)$ e as meninas $71,2(D P=5,4)$. 0 valor mínimo atingido pelos meninos foi de 55 e o valor máximo foi de 77 pontos. As meninas, por sua vez, alcançaram o mínimo de 59 e o máximo de 81 pontos. Visando verificar a diferença entre os meninos e as meninas na pontuação total da escala do ponto de vista estatístico, realizou-se um teste $\mathrm{t}$ de Student, o qual revelou que, embora tenha havido uma diferença nas médias alcançadas pelos referidos grupos, ela não foi estatisticamente significante $(\mathrm{t}=2,59 ; \mathrm{p}=0,01)$.

As pontuações nas subescalas estratégias cognitivas, estratégias metacognitivas e ausência de estratégias também foram levantadas e verificou-se que apesar da sutil diferença na pontuação das subescalas em favor das meninas, em especial na subescala de ausência de estratégias, o teste $t$ de Student indicou que não havia significância estatística, como pode ser visto na tabela 1 .

\begin{tabular}{|c|c|c|c|c|}
\hline Subescalas & $\begin{array}{c}\text { Média e DP } \\
\text { Meninos }\end{array}$ & $\begin{array}{l}\text { Média e DP } \\
\text { Meninas }\end{array}$ & $\mathbf{t}$ & $\mathbf{p}$ \\
\hline $\begin{array}{l}\text { Estratégia } \\
\text { Cognitiva }\end{array}$ & $23,1 \pm 3,7$ & $23,8 \pm 3,0$ & 0,84 & 0,70 \\
\hline $\begin{array}{l}\text { Estratégia } \\
\text { Metacognitiva }\end{array}$ & $16,0 \pm 1,8$ & $16,6 \pm 2,0$ & 1,32 & 0,61 \\
\hline $\begin{array}{l}\text { Ausência de } \\
\text { Estratégias }\end{array}$ & $28,4 \pm 4,9$ & $30,6 \pm 4,3$ & 1,91 & 0,06 \\
\hline
\end{tabular}


Quando analisadas as estratégias individualmente, pode-se perceber que a estratégia mais utilizada pelos participantes foi uma estratégia metacognitiva (item 18). Nesse item, a maioria dos meninos $(73,5 \%)$ reportou olhar as questões erradas sempre que recebem uma nota e $79,4 \%$ das meninas afirmaram o mesmo. Outra estratégia bastante citada, também metacognitiva, refere-se à compreensão de leitura (item 27), em que 70,6\% dos meninos e $73,5 \%$ das meninas afirmaram que quando não entendem a leitura, normalmente param e leem novamente.

Algumas estratégias foram utilizadas de modo semelhantes pelos meninos e pelas meninas. Cerca de $52,9 \%$ dos alunos disseram nunca desistir quando uma tarefa é difícil ou chata (item 7) e 41,2\% disseram que não costumam ficar se levantando toda hora para pegar algum material depois que se sentam para fazer o dever de casa (item 23). Além disso, 61,8\% afirmaram que sempre costumam fazer questões para si mesmos quando terminam de estudar para uma prova (item 10) e 50\% disseram que às vezes costumam se distrair ou pensar em outra coisa quando estão lendo (item 26).

Outras estratégias, contudo, foram utilizadas de forma diferente pelos meninos e pelas meninas. Cinquenta por cento dos meninos e $26,5 \%$ das meninas afirmaram nunca fazer uma lista de ideias antes de começar a escrever uma redação (item 2). Cinquenta por cento dos meninos e $35,3 \%$ das meninas disseram que às vezes fazem esquemas usando as ideias principais do texto (item 9). Em outro item, $41,2 \%$ dos meninos e $70,6 \%$ das meninas disseram relacionar os conteúdos novos com o que já sabiam (item 16). Ainda, 76,5\% dos meninos e $32,4 \%$ das meninas disseram que às vezes se esquecem de fazer o dever de casa (item 28).

Analisando as subescalas separadamente, pode-se perceber que na subescala de estratégias cognitivas, os meninos referenciaram utilizar uma parcela considerável das estratégias, às vezes $(1,5,9,11$, 14 e 16), de modo tal que apenas três estratégias foram citadas pela maior parte como sendo usadas sempre (10,17 e 20) e duas estratégias, nunca (2 e 4). De modo muito semelhante, as meninas afirmaram utilizar boa parte das estratégias cognitivas às vezes $(1,2,5,11,14,16$ e 20). Somente três estratégias foram citados pela maior parte como sendo usadas sempre $(9,10$ e 17) e uma nunca (4) foi utilizada pela maioria das participantes.
No que concerne as estratégias metacognitivas, percebeu-se que a maior parte dos meninos reportou utilizar as estratégias apenas às vezes $(6,13,22$, 29 e 31). Poucas estratégias foram referenciadas como sendo utilizadas sempre (18 e 27) pela maioria dos participantes. As meninas, de modo similar, reportaram usar às vezes quatro estratégias $(6,13$, 22 e 29) e sempre, três delas (18, 27 e 31 ).

No que diz respeito à subescala ausência de estratégias, observou-se que sete estratégias de aprendizagem foram referenciadas pelos meninos como sendo utilizadas às vezes $(3,12,15,21,25,26$ e 28) e seis como nunca usadas $(7,8,19,23,24$ e 30 ). De modo um pouco diferente, as meninas reportaram utilizar às vezes apenas quatro estratégias $(12,21,26$ e 30$)$ e nunca nove estratégias $(3,7$, $8,15,19,23,24,25$ e 28 ).

\section{Discussão}

Analisando a pontuação total da escala, percebe-se que as meninas alcançaram pontuações mais elevadas. Tal dado foi encontrado por diversos pesquisadores (Cruvinel, 2003; Gomes, 2002; Lins, Araujo, \& Minervino, 2009, 2011; Oliveira, 2008; Oliveira, Boruchovitch, \& Santos, 2011), embora ainda não seja um consenso os motivos que levam a essa diferenciação. Desse modo, alguns supõem que essa diferença ocorre por questões inerentes ao desenvolvimento humano, uma vez que as meninas amadurecem antes que os meninos (Fernandes, 2010). Nesse caso, pode-se pensar que as meninas, por se desenvolverem social, emocional e mentalmente mais cedo, podem usufruir do entendimento adquirido acerca das estratégias de aprendizagem com mais eficácia.

No que concerne as estratégias mais utilizadas, percebe-se que tanto os meninos como as meninas reportaram utilizar com frequência duas estratégias metacognitivas. De modo semelhante, Lins, Araujo e Minervino (2009; 2011) encontraram que as estratégias mais referenciadas pelos participantes foram as metacognitivas. Dessa forma, supõe-se que os estudantes percebem com facilidade quando estão com dificuldades para aprender, contudo, em decorrência da maioria das estratégias serem referenciadas como sendo utilizadas apenas às vezes, pode-se questionar se eles, percebendo as dificuldades em seu processo de aprendizagem, modificam suas

Psicol. Argum. 2014 jul./set., 32(78), 9-15 
estratégias de modo a alcançar resultados mais significativos. Nesse sentido, Costa (2000) pontua que o fato dos estudantes reportarem utilizar estratégias de aprendizagem não implica que eles necessariamente o façam com frequência, podendo inclusive ter respondido positivamente as questões propostas por entenderem que tais respostas eram esperadas, desejáveis socialmente.

Percebeu-se ainda que muitas estratégias são usadas de modo parecido tanto pelos meninos como pelas meninas, indicando que embora existam diferenças entre os grupos, algumas foram aprendidas de modo similar. Ou seja, os estudantes, independentemente do gênero, possuem mecanismos que os levam a desenvolver métodos de aprendizagem semelhantes.

Contudo, observou-se que os estudantes reportaram utilizar com frequência diferente algumas estratégias, demonstrando que mesmo tendo aprendido a utilizar as estratégias de modo similar, as meninas parecem compreender a eficácia delas para a aprendizagem, uma vez que as utilizam mais frequentemente. Nesse sentido, autores como Silva e Sá (1997) pontuam que os estudantes precisam compreender o porquê de se utilizar as estratégias de aprendizagem, pois caso não tenham essa compreensão as utilizarão pouco ou não o farão.

No que concerne as subescalas, percebeu-se que nas de estratégias cognitivas, os participantes responderam de modo parecido, havendo pequenas diferenças quanto a frequência de algumas estratégias, indicando que ambos os grupos usam estratégias de ensaio, de elaboração e de organização semelhantemente. As estratégias cognitivas de ensaio são as mais simples, geralmente utilizadas pelos alunos mais novos. As estratégias de elaboração, por sua vez, são mais elaboradas, características de estudantes mais estratégicos. As estratégias de organização são utilizadas para estruturar o material a ser aprendido e podem maximizar o entendimento, na medida em que sua utilização implica que os estudantes saibam selecionar tanto os aspectos principais como os detalhes dos textos (Boruchovitch, 1999; 2001).

No que diz respeito às estratégias metacognitivas, ambos os grupos reportaram utilizar-se de modo semelhante, indicando que tanto as estratégias de regulação como as de monitoramento são usadas por eles. De acordo com Boruchovitch (1999; 2001), ao usar estratégias de monitoramento, os educandos demonstram estar conscientes do quanto estão sendo capazes de captar e absorver do conteúdo que está sendo ensinado, e ao utilizar as de regulação revelam saber checar as falhas na compreensão e modificar as estratégias a fim de alcançar os objetivos inicialmente propostos.

Quando analisada a subescala ausência de estratégias, percebeu-se que as meninas utilizam um número maior de estratégias, demonstrando um comportamento menos desadaptativo. Assim, imagina-se que a diferença na pontuação geral na escala não se deve ao uso de estratégias cognitivas e metacognitivas, uma vez que as diferenças nesse repertório forma mínimas, mas a falta de comportamentos considerados disfuncionais no momento do estudo por parte do grupo feminino.

\section{Considerações finais}

Pelo exposto, percebe-se que as diferenças entre meninos e meninas já verificadas em estudos da região sudeste, podem se confirmar em outras regiões do país, indicando que embora existam diferenças culturais e sociais, o sexo feminino, de modo geral, se sobressai no início da escolarização formal. Ao analisar mais especificamente a diferença no repertório de estratégias de aprendizagem de meninos e meninas, percebeu-se que a diferenciação entre esses parece se efetivar quando do uso de comportamentos disruptivos, uma vez que ambos os grupos demonstraram utilizar basicamente as mesmas estratégias, sendo a diferença observada na ausência de comportamentos considerados indesejáveis por parte do grupo feminino.

Sabe-se que os dados obtidos no presente estudo não podem ser considerados conclusivos, uma vez que o grupo amostral é restrito a poucos alunos, mas acredita-se que trazem resultados interessantes, na medida em que confirmam algumas diferenças entre a frequência de algumas estratégias de aprendizagem quando considerado o gênero, o que já vem sendo discutido entre os pesquisadores da área. Nesse sentido, novas investigações com uma amostra maior precisam ser realizadas visando explorar melhor possíveis diferenças entre esses grupos na pontuação da escala de estratégias de aprendizagem e, principalmente, o que leva a essa diferenciação. Nesse caso, pode-se inclusive utilizar medidas comparativas como, por exemplo, as notas dos estudantes, para que se possa comparar o uso das estratégias com os resultados obtidos em seu uso frequente. 


\section{Referências}

Boruchovitch, E. (1999). Estratégias de Aprendizagem e Desempenho Escolar: Considerações para a Prática Educacional. Psicologia: Reflexão e Crítica, Porto Alegre, 12(2), 361-376.

Boruchovitch, E. (2001). Algumas Estratégias de Compreensão em Leitura de Alunos do Ensino Fundamental. Psicologia Escolar e Educacional, Campinas, 5(1), 19-25.

Boruchovitch, E., Santos, A. A. A., Costa, E. R., Neves, E. R. C., Cruvinel, M., Primi, R., \& Guimarães, S. E. R. (2006). A construção de uma Escala de Estratégias de Aprendizagem para Alunos do Ensino Fundamental. Psicologia: Teoria e Pesquisa, Brasília, 22(3), 297-304.

Costa, E. R. (2000). Estratégias de aprendizagem e a ansiedade de alunos do ensino fundamental: implicações para a prática educacional. Dissertação de Mestrado. Faculdade de Educação, Universidade Estadual de Campinas. Campinas.

Cruvinel, M. (2003). Depressão infantil, rendimento escolar e estratégias de aprendizagem em alunos do ensino fundamental. Dissertação de mestrado. Faculdade de Educação, Universidade Estadual de Campinas. Campinas.

Cruvinel, M., \& Boruchovitch E. (2004). Sintomas depressivos, estratégias de aprendizagem e rendimento escolar de alunos do ensino fundamental. Psicologia em Estudo, 9(3), 369-378.

Dembo, M. H. (1994). Applying educational psychology (5 Ed.). New York: Longman Publishing Group.

Fernandes, V. (2010). Diferenças entre meninos e meninas na escola. Gazeta do povo. Recuperado de http:// www.gazetadopovo.com.br/opiniao/conteudo. phtml?id=1026012

Gomes, M. A. M. (2002). Aprendizagem auto-regulada em leitura numa perspectiva de jogos de regras. Dissertação de mestrado. Faculdade de educação, Universidade Estadual de Campinas. Campinas.
Gomes, M. A. M., \& Boruchovitch, E. (2005). Desempenho no Jogo, Estratégias de Aprendizagem e Compreensão na Leitura. Psicologia: Teoria e Pesquisa, 21(3), 319-326.

Lins, M. R. C., Araujo, M. R., \& Minervino, C. A. S. M. (2009). Estratégias de aprendizagem em crianças do ensino fundamental. Monografia (Trabalho Acadêmico Orientado). Centro de Ciências Biológicas e da Saúde, Universidade Estadual da Paraíba, Campina Grande, PB.

Lins, M. R. C., Araujo, M. R., \& Minervino, C. A. S. M. (2011). Estratégias de aprendizagem empregadas por estudantes do ensino fundamental. Psicologia Escolar $e$ Educacional, 15(1), 63-70.

Oliveira, K. L. (2008). Escala de estratégias de aprendizagem para o ensino fundamental: análise de suas propriedades psicométricas. Tese de Doutorado. Faculdade de Educação, Universidade Estadual de Campinas. Campinas.

Oliveira, K. L., Boruchovitch, E., \& Santos, A. A. A. (2011). Estratégias de aprendizagem no ensino fundamental: análise por gênero, série escolar e idade. PSICO, 42(1), 98-105.

Ribeiro, C. (2002). Aprender a Aprender: Algumas Considerações Sobre o Ensino de Estratégias de Estudo. Máthesis, 11, 273-286.

Silva, A. L. \& Sá, I. (1997). Saber estudar e estudar para saber. Coleção Ciências da Educação. Porto, Portugal: Porto Editora.

Weinstein, C., \& Mayer, R. (1983). The Teaching of Learning Strategies. Innovation Abstracts, 5(32).

Zimmerman, B. J., \& Martinez-Pons, M. (1988). Construct Validation of a Strategy Model of Student Self-Regulated Learning. Journal of Educational Psychology, 80(3), 284-290.

Psicol. Argum. 2014 jul./set., 32(78), 9-15 